\title{
FLOWS, FLARES, AND FORMATION OF UMBRAE AND LIGHT BRIDGES IN BBSO REGION No. 1167
}

\author{
HAROLD ZIRIN and HAIMIN WANG \\ Big Bear Solar Observatory, California Institute of Technology, Pasadena, CA 91125, U.S.A.
}

(Received 30 June, 1989; in revised form 28 July, 1989)

\begin{abstract}
We present high-resolution observations of the large active region BBSO No. 1167 (Boulder No. 5060) which cast new light on the structure of sunspot regions. We obtained excellent data, highlighted by videomagnetograms (VMG) obtained with our $65-\mathrm{cm}$ telescope, which give unprecedented spatial resolution, about $0.5^{\prime \prime}$ for much of two 11-hr periods. This permitted us to see details of the field evolution and flows in the AR. The $\mathrm{H} \alpha$ filtergrams and D3 filtergrams permit study of these magnetic changes compared to spots and chromospheric structure.

The region was a huge but simple active region (CMP July 2, 1988) in which we observed rapid flux emergence for several days. Because the new flux generally matched the old, there were few large flares. However, there were 14 flares on June 28 and 29 , mostly in two sites. The first site was a $\delta$ spot which already existed when the active region appeared on the east limb. This site showed little change of magnetic structure during our observing period. The second site is an area disturbed by new flux emergence, which included a $\delta$ spot which formed and disappeared in two days, and a rapidly moving $p$ spot. Flares ocurring at one site almost always produced footpoints at the other. The delay between flash phases of the same flare at the two sites ranges from 40 to $160 \mathrm{~s}$.

The magnetograms show complex fine structure, with some closely interwined regions of opposite polarity. In a region of new flux emergence, positive (leading polarity) flux flows along elongated channels immersed in the negative flux. Moving magnetic features occur around all of the spots.

We point out other interesting aspects of this large region: (1) While there is extensive penumbra around the main umbrae, there is also significant penumbra apparently unrelated to any spot. These unusual penumbrae are either due to flux returning to the surface, flux left behind by the moving umbra, or associated with pores that appear and disappear. (2) We observed umbrae to move faster than the accompanying penumbrae, and concluded that penumbrae are not a simple extension of the umbra. (3) We found that combining spots of the same polarity do not completely merge, but are always separated by a thin light bridge. This means that the emerging flux loops are discrete entities.
\end{abstract}

\section{Introduction}

The huge active region BBSO No. 1167 appeared on the east limb on June 24, 1988 and was followed by us until July 5 under generally good conditions. A fairly complete data set was obtained at Big Bear Solar Observatory (BBSO) on this region. The development of the region from June 27 to July 2 can be seen in Figure 1.

When first photographed on June 25 the region had only the big $p$ spot $p 1$ and a cluster of $f$ spots, but on June 26 several EFR's emerged on the upper (N) side, and Figure 1 shows how their rapid westward motion led to their merging into a second large $p$ spot which moved ahead of $p 1$ to the upper right corner in Figure 1. Despite its size $\left(3 \times 10^{-3}\right.$ of the hemisphere) the overall magnetic structure of the region was simple and it had few flares. During the peak of the flux emergence there was modest flare activity on June 28 and 29 associated with the formation and disappearance of a $\delta$ spot and an anomalous leader spot. In this paper we have concentrated on: 

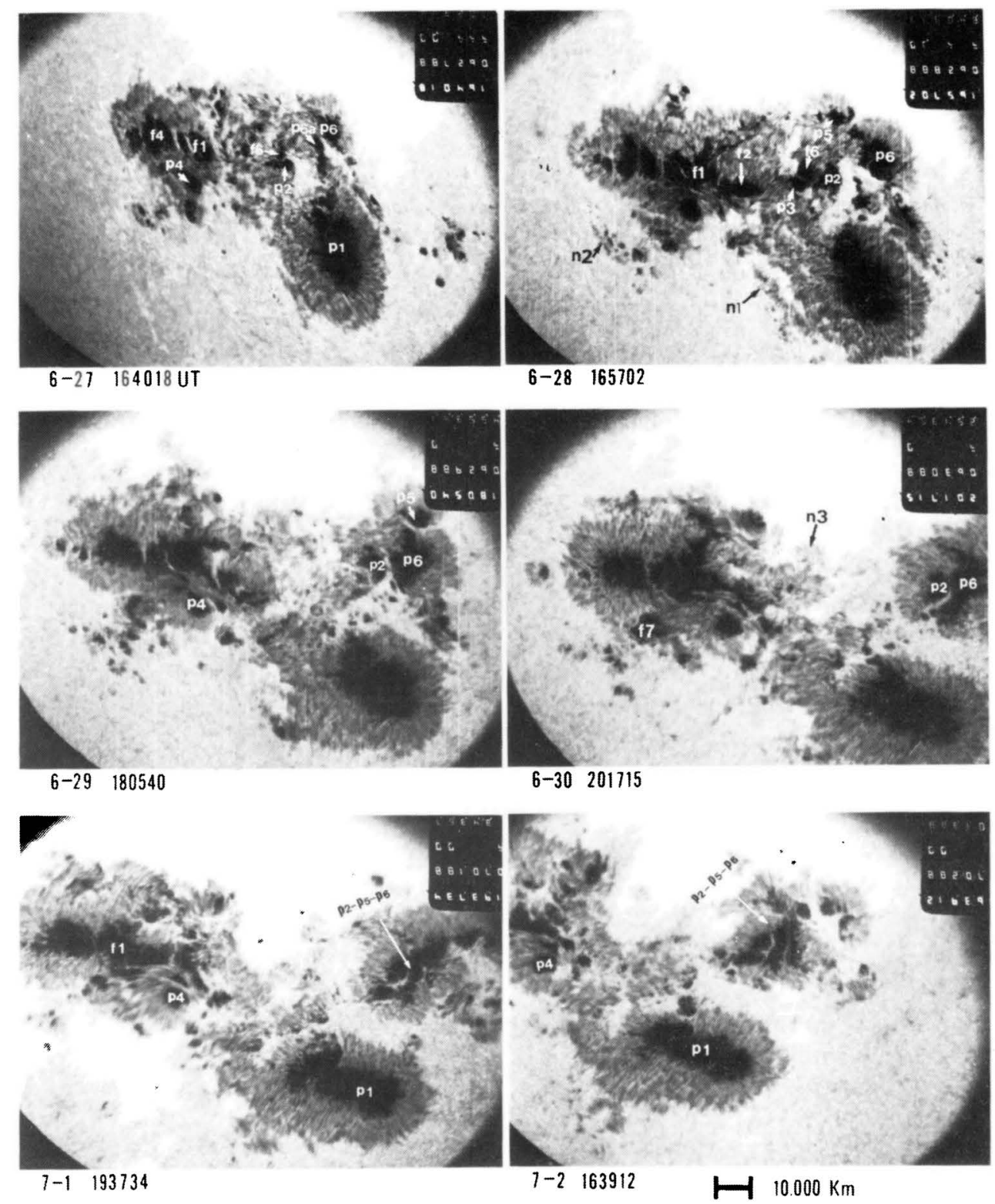

Fig. 1. D3 images for June 27-July 2, 1988, showing the evolution of the group. Sunspots are marked either $p$ or $f$ according to their polarities. All pictures are $\mathrm{N}$ top, $\mathrm{W}$ right. The facular brightness is somewhat reduced in this line by overlying absorption.

(1) Morphology changes and flows of sunspots and magnetic structures.

(2) Properties of growing spots.

(3) $\delta$ spots.

(4) The location and occurrence of flares.

The combination of high time and spatial resolution observations with a big sunspot gives an exceptional chance to study these problems. By rapid projection of re-registered digital images we could detect small flows and link them to their sources. Despite some claims to the contrary, all the larger scale motion of magnetic elements in an active region is due either to sunspot separation associated with flux emergence or to Evershed flow, including the outward flow of moving magnetic features (MMF) in the surrounding 
penumbra. The former results in rapid westward movement of $p$ spots, which in this case is such as to merge them all together, but which, if the motion is into opposite polarity, can lead to flares. On a small scale there is more varied motion. In particular, elongated channels of $p$ polarity trail the moving $p$ spots and are filled with elements of flux moving along the channel.

The changes observed in the magnetic fields are mostly the predictable ones associated with flux emergence. But we observed an excellent example of the disappearance of two spots of opposite polarity locked in a $\delta$ spot on 28-29. In addition, particularly fine observations of spot formation by convergent flow were obtained, as well as motion of umbrae relative to the penumbrae in which they lie.

As usual, all of the flares in the region are associated with neutral lines. Most are associated with the $\delta$ spot or the motion of a $p$ spot past $f$ polarity, but a number are associated with a stable satellite spot which shows little change.

The merging of the leader spots of the different EFR's is one of the most interesting phenomena observed. During this process we see how the near-merging of umbrae of the same polarity leads to the formation of a light bridge and the penumbra changes its form in response to outside fields.

\section{Observation}

Observations include magnetograms, white light, $\mathrm{H} \alpha$ center line and off band filtergrams, and D3 filtergrams. However, while frequent VMG's were recorded on film, digital VMG recording was limited to two per hour because of tape problems.

The active region (BBSO No. 1167) was followed immediately after it appeared from the east limb on June 24,1988 , until July 5 . Flux emergence which began June 26 peaked on June 28 and 29 , causing a peak in flows and flares. So our study was concentrated on these days. Eleven hours of data were obtained on each day.

In order to achieve higher spatial resolution, the VMG system (Mosher, 1976; Zirin, 1985) was put in the center bench of $65-\mathrm{cm}$ vacuum reflector at BBSO, instead its normal position on the $25-\mathrm{cm}$ telescope. This gave a substantial improvement in sensitivity and resolution during the best conditions. The image scale of the magnetograms in $0.3^{\prime \prime}$ pixel $^{-1}$, giving the overall field of $160^{\prime \prime}$ by $160^{\prime \prime}$. Simultaneously, D3, center line $\mathrm{H} \alpha$ and $\mathrm{H} \alpha+0.7 \AA$ filtergrams were obtained with the east bench of the 65-cm telescope, east and west bench of the 25-cm telescope, respectively. The field of the $25-\mathrm{cm}$ telescope at BBSO is $350^{\prime \prime}$ by $300^{\prime \prime}$.

Magnetograms were re-registered by applying the maximum correlation technique (Wang and Zirin, 1988). The saturation contours required by the limitations of 8-bit tape storage are then removed with a special unwrapping program. The geometric foreshortening was corrected according to the coordinates of the region on the solar disk. The images are displayed as a movie at an acceleration rate of $1: 10000$. Prints made from the film are the best way to show the $\mathrm{H} \alpha$ images, since they have the best spatial resolution and intensity range. However, it is difficult to manipulate these images without digitizing them, so we did not correct the geometric foreshortening for these 
images. Therefore, we display uncorrected magnetograms which can be scaled to the same size as the $\mathrm{H} \alpha$ images. Because the D3 images from the $65-\mathrm{cm}$ telescope cover a more limited area, they may be digitized with less resolution loss. So for each observing day, 60 D3 images were digitized and re-registered. D3 movies show the foot points of larger flares.

In addition to the movies, selected magnetograms, $\mathrm{D} 3, \mathrm{H} \alpha$, and white-light pictures were printed at the same spatial scale. Initial brightening patches of flares, flare foot points, and maximum brightening pattern of flares were located and drawn on the magnetograms.

\section{Morphology and Flows}

A very large bipolar spot appeared at the $\mathrm{E}$ limb on June 24, a day when we were occupied with intense activity in a region at the $\mathrm{W}$ limb. This region was young, having appeared on the back side of the Sun. However, major growth had ceased by June 28. Figure 2 shows magnetograms and the corresponding D3 images for June 28 and 29,
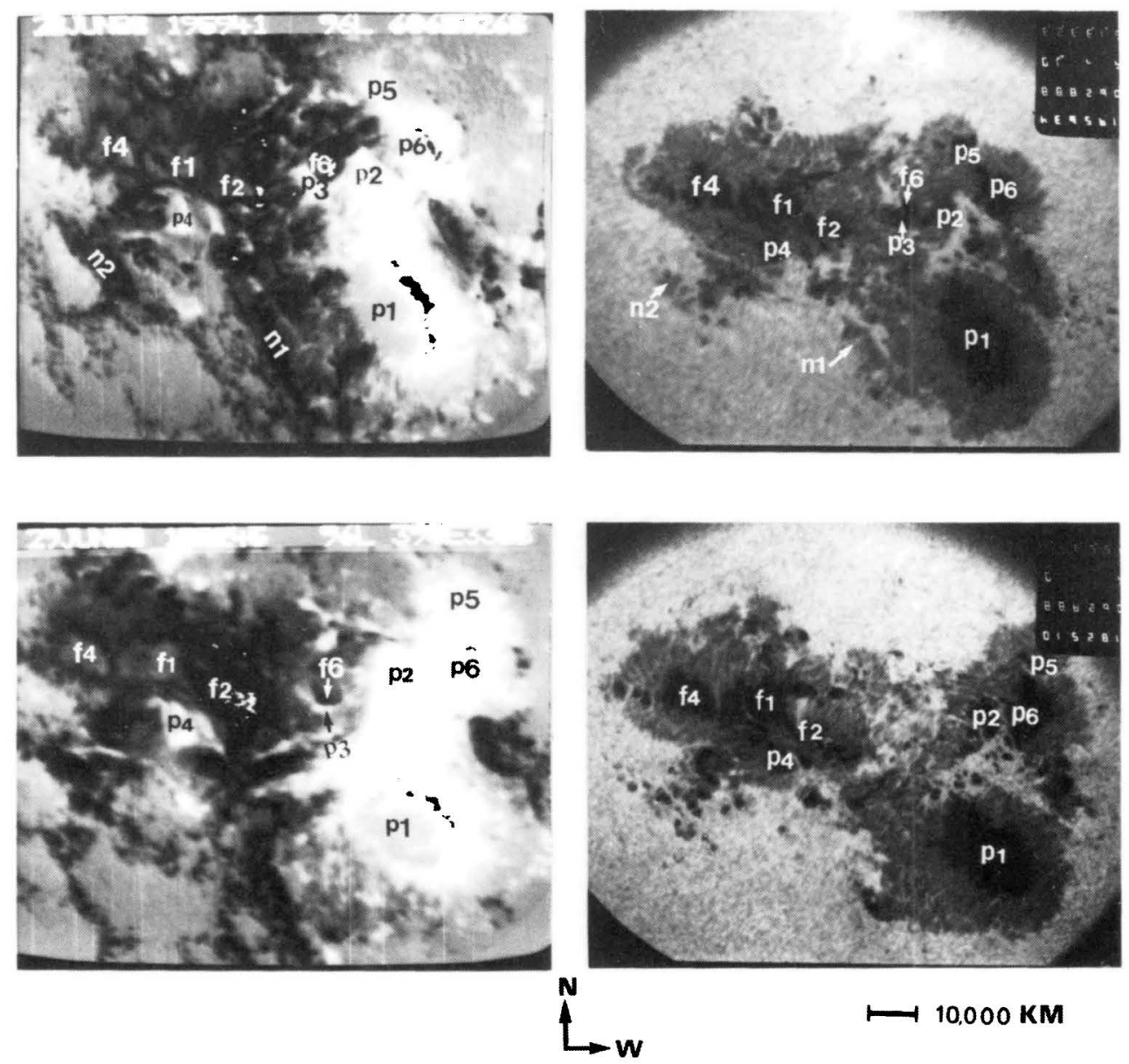

Fig. 2. Magnetograms and corresponding D3 images for June 28 and June 29, 1988. Note that the field in the trailing penumbra of $p 1$ changes apparent sign from the 28th to 29th as the spot moves on to the disk. The field is actually positive (white), but the limbward penumbra appears dark as the field lines turn away from us. 
respectively. Sunspots are marked as either $p$ (proceeding) or $f$ (following) according to their polarities. The gray area inside each sunspot is due to the low brightness of the spot umbrae, which places them below the brightness threshold of the VMG A/D conversion system. Thus with these settings there is no signal from the umbra.

The region had a big stable sunspot ( $p 1)$ of positive magnetic polarity, which may be seen near the S-W corner of the field of view. The following counterpart was a cluster of umbrae $f 1, f 2$, and $f 4$. In addition, an extensive penumbral area was associated with the group, some parts of which (marked $n 2$ ) were not clearly associated with umbrae.

Higher spatial resolution and faster movie running speed enhance the apparent velocities of sunspots and surrounding plage magnetic elements. We used these to pick out the important moving features, for which vectors are given in Figure 3. The most striking material motion is rapid westward flow of the growing leader spots $p 2, p 5$, and p6. $p 5$ (Figures 1 and 2) moved westward at a speed of $0.4 \mathrm{~km} \mathrm{~s}^{-1}$ on June 28 and it, along with the satellite spot $p 4$, was responsible for the most flares. This can be seen in Figure 1, where $p 5$ was E of $p 6$ on the 28th and moved $\mathrm{W}$ of it by the 29th. The umbra of the spot was not visible on June 27. The speed of $p 5$ on the 28th decreased to less than $0.1 \mathrm{~km} \mathrm{~s}^{-1}$ on June 29, when the penumbra of the spots became well formed.
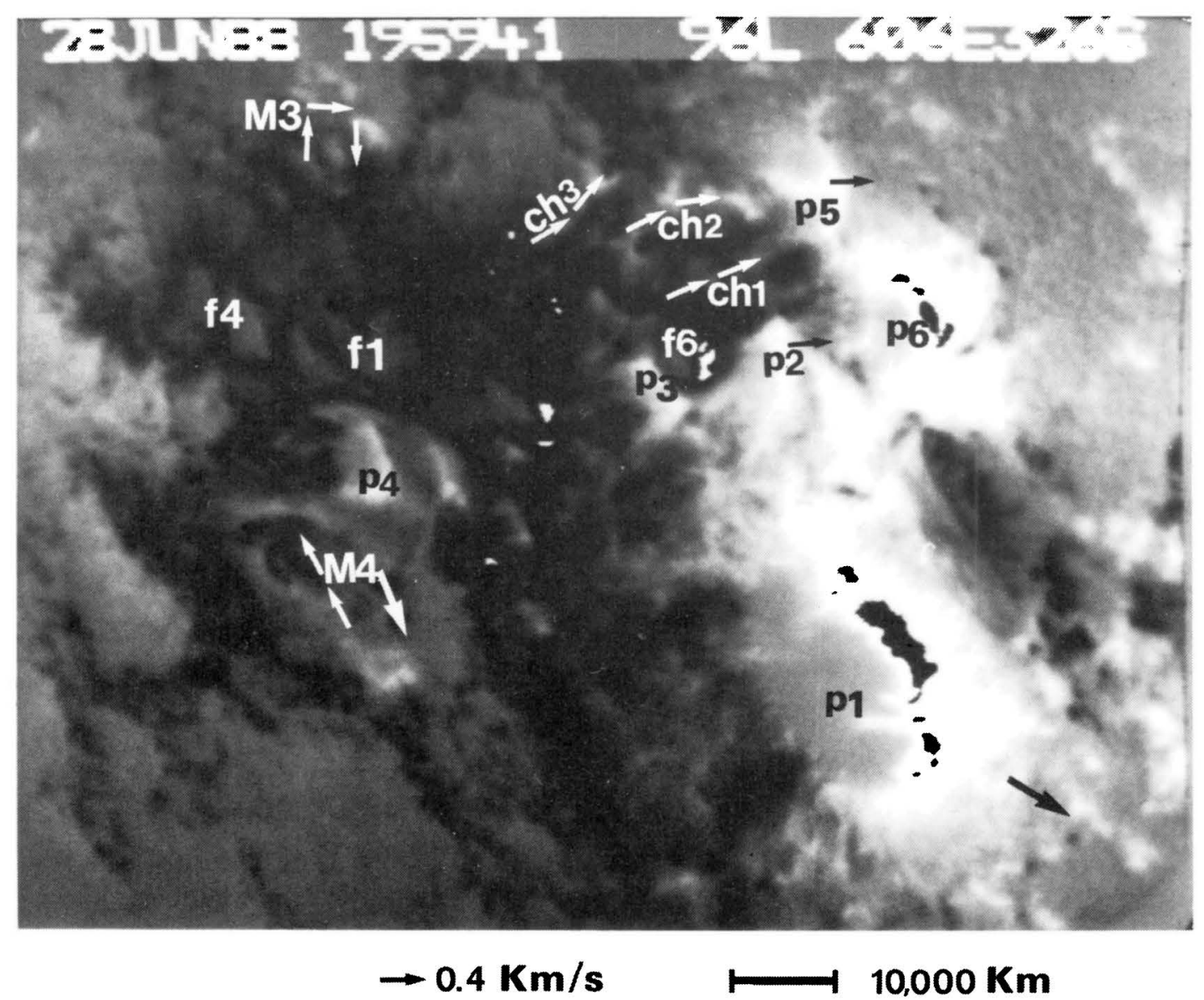

Fig. 3. Flow vectors showing prominent mass motions on June 28 in the active region. The image and the velocity amplitude scale are marked at the bottom. 
Within the new EFR (the moving spot $p 5$ and its following partners) the magnetic fields show complicated structure and velocity patterns. While lower resolution VMG's show such EFR's as simple bipolar, the present data show the following portion, at least, to be highly mixed polarity. This is best seen in Figure 6 (top) where we see the arch filaments going from a fragmented $p 5$ to the following components of the EFR, which are in the area marked ch2 and ch3 in Figure 3. Mixed polarities of this sort appear in the data of Tarbell et al. (1988) who observed relative motion of opposite polarities over a 30-min baseline. With the present data, which permits measurement over a $10-\mathrm{hr}$ baseline, we can detect substantial motion of features in elongated $p$-polarity channels (ch1-3) immersed in larger areas of opposite polarity and elongated in the direction of motion. Flows in ch1 and ch2 move towards the right, and merge to the moving spot, while ch3 moves $\mathrm{N}$, cancelling with negative flux there. All the positive flux elements are moving with a speed of about $0.4 \mathrm{~km} \mathrm{~s}^{-1}$. This is typical EFR behavior, the $p$ spot

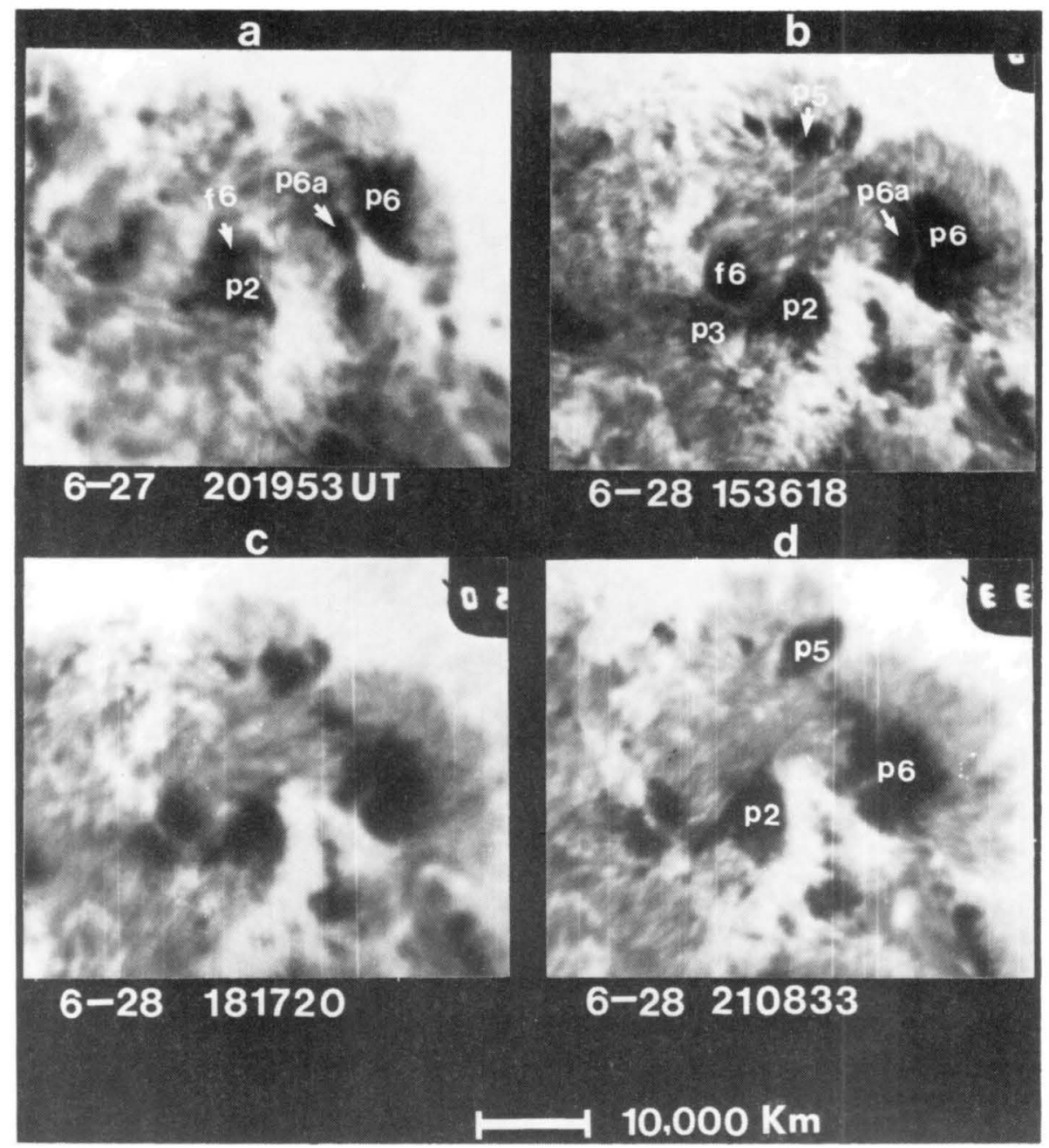

Fig. 4. Rapidly changing spots: (a) $p 6 a-f 6$ is forming. (b) At the top, the spot $p 5$ forms from a cluster of pores. Below, $p 6 a-f 6$ grows rapidly until $p 6 a$ merges into $p 6$ and $f 6$ forms a $\delta$ spot with $p 3$, which is rapidly separating from $f 2$. The two spots lock together and one sees sheared penumbra in between. (c) The pores merge to form $p 5$. (d) $p 5$ moves $\mathrm{W}$ to merge with the spot in the front of the penumbra. $p 2$ begins to move $\mathrm{W}$ rapidly and we already see the shrinking of the $\delta$ spot. 
moving westward while the $f$-polarity is stationary. Near the moving spot $p 5$, some $f$-polarity negative elements move with the spot, and eventually merge into it, cancelling with the positive flux of the moving spot.

In Figures 4 and 5 we show the formation of $p 5$ on June 28 from a cluster of small pores. In contrast to the formation reported by Zirin (1987), where the spot forms by gradual filling in of the space between pores, here the spots flow together in $2 \mathrm{hr}$ to form a substantial spot, as first observed by Vrabec (1974). However, then the new spot continues to flow forward relative to the penumbra until it reaches the front edge of the penumbra and merges with an umbra there. So here the penumbra is not simply the diverging part of the umbra, but bears a more complex relation to it. Certainly in the W-direction the field lines are swept up by the moving umbra and are probably vertical. Normally we assume that the penumbra is intrinsically connected to the umbra and moves with it; this is the first reported case of umbral motion relative to the penumbra. Whether this truly is relative motion or just a change of shape on the $p$ side of the spot cannot be determined. However, it shows one way that a spot with penumbra missing on one side may form.

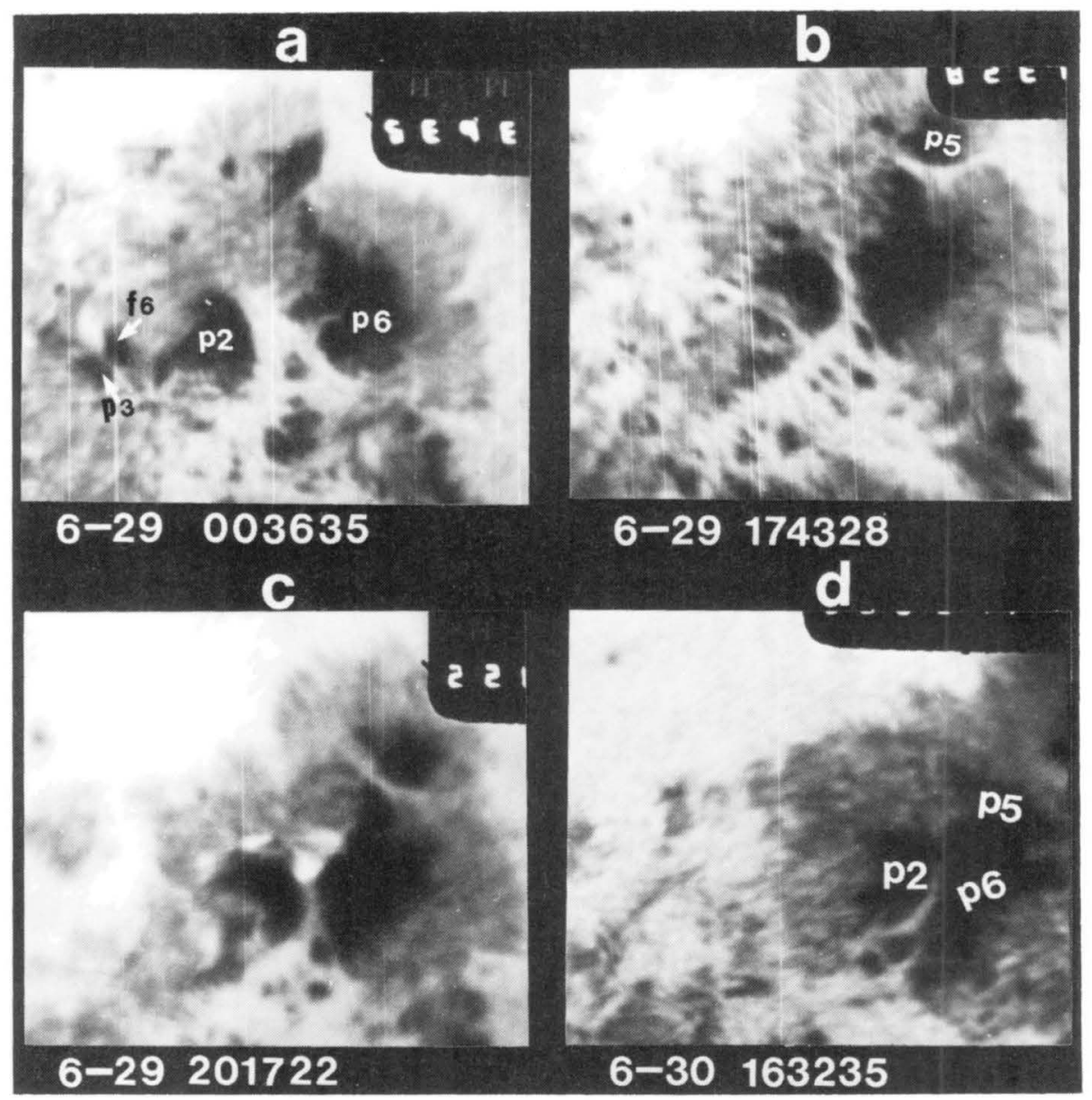

Fig. 5. Further evolution. (a) The $\delta$ spot shrinks. (b) $p 5$ moves around to the front of $p 6$, rotating $90^{\circ}$ and forming a light bridge. $p 2$ reaches $p 6$ and forms a light bridge. The $\delta$ spot has disappeared. (c) Footpoints of a flare that began near $p 4$ appear between $p 2$ and $p 6$. (d) Spots $p 2, p 5$, and $p 6$ have merged, but the light bridges remain. 
Another important motion was displayed by spot $p 2$, which formed with $f 2$ on June 27 and moved ahead to merge with spot $p 6$. As with $p 5$, there was no penumbra in the direction of umbral motion, again indicating that the normally diverging penumbral field lines are either swept aside or forced into a vertical direction by the approach of like fields. These two spots showed the largest approaching motion $\left(0.2 \mathrm{~km} \mathrm{~s}^{-1}\right)$ at the beginning of the period, probably due to the recent formation of $p 2$.

In contrast to $p 5$, which pushed through opposite polarity and produced activity, $p 2$ pushed into similar polarity to merge with it. Normally this kind of field rearrangement does not give much activity, because no sheared neutral line is formed. In this case, emission from one substantial flare appeared between the merging spots although this may have been a secondary set of footpoints.

Other flows are marked in Figure 3. M3 was an area of curved, almost vortical flow around some satellite pores. On the E there was a strong Evershed flow out of a large $f$ spot, which led into an inward flow on the $\mathrm{W}$ side of these spots into the main spot. In $\mathbf{H} \alpha$, we see a circular bright area.

$M 4$ is another area with prominent flow motion. There are two flow components: in the left part, negative magnetic fields move upwards, they are pushed into a piece of positive flux; on the right, some flux elements move $S$ with a speed of nearly $1 \mathrm{~km} \mathrm{~s}^{-1}$.

On June 29, flows which were prominent in the previous day had almost disappeared. The most obvious motions on that day are the outflows from spots, i.e., MMF's. They are observed in every field-free area near every spot in the group, filling out all the possible spaces outside the penumbrae of spots. The speed of all the moving magnetic features are from 0.5 up to $1 \mathrm{~km} \mathrm{~s}^{-1}$. The most prominent ones are visible ahead of spot $p 1$, at the lower right of Figure 3. Whether the MMF's also occur in regions of strong field adjoining the penumbrae cannot be determined. We find in studying active regions in general that MMF's are present around every spot with penumbra.

\section{Light Bridges and Peculiar Penumbrae}

Bray and Loughhead (1964) give fine examples of the formation of light bridges during the breakup of large, complex umbrae. Cases are given by Hale and Nicholson (1938). Zirin and Tanaka (1981) show another, involving the expulsion of an umbra of one polarity from a $\delta$ configuration. But the present data show that, not surprisingly, light bridges can form as umbrae merge, just as well as when they split. This is quite clear in the merging of $p 2-5-6$, which leads to a cluster of umbrae separated by narrow light bridges. $p 6$ is already composed of two umbrae that merged on the 27th, and $p 2-5-6$ never really merge. Starting on July $1, p 2-5-6$ started to break away (see the bottom 2 frames of Figure 1) from the rest of the spot group.

An overexposed frame shows that the big umbra $p 1$ is in fact formed of several distinct umbrae. We believe that if we had proper images of the examples shown by the authors quoted above, we should see them to be quite distinct umbrae, and the subsequent expansion of the light bridge takes place as shown by Zirin and Tanaka (1981). This 
implies that the individual umbrae remain distinct inside the big spots for much of their lifetime, although in the final decay they may truly merge.

Penumbrae are the transition between the umbrae and photosphere. They are normally defined as the outer part of sunspots where the brightness is between umbra and photosphere and the diverging magnetic force lines are parallel to the surface. Danielson (1961) proposed that penumbrae are a qualitatively different convective regime appropriate to horizontal field. Our images, and in fact those of many active regions show penumbrae (e.g., $n 1$ and $n 2$ in Figure 1) apparently independent of umbrae. In each case we observe longitudinal field, but the absence of vector information makes it difficult to decide the field configuration. Their sources are different. Careful comparison with the $\mathrm{H} \alpha$ images in Figures 6 and 7 shows that the isolated penumbra $n 1$ is the point where flux returns to the surface, and the magnetograms show the polarity to be reversed. Apparently the flux loops are so tightly contained that they can affect the photospheric brightness. So this is a new kind of penumbra associated with re-entrant fields.

When we view the accelerated continuum movies, we see that the umbrae always move faster than the penumbrae. This has already been referred to for $p 5$, but even occurs for small pores, such as those near $n 3$. These pores move $0.3-0.4 \mathrm{~km} \mathrm{~s}^{-1}$, while the penumbra moves $0.1-0.2 \mathrm{~km} \mathrm{~s}^{-1}$. The pores which can be seen in Figure 1 have moved out ahead of penumbra $n 3$, and they eventually reach the penumbra behind $p 5$. Because umbrae are always the fast moving elements, we think that the more diffuse and extensive penumbra simply faces more drag as it tries to keep up. Other peculiar penumbrae, such as $n 2$, appear to be associated with pores that appear and disappear. Of course, it is possible that Danielson's roll convection is still going on in these regions, but it is clear that penumbra does not always fit the usual definition.

If we compare the magnetograms of June 28 and June 29 (Figure 2), we see that the part of penumbra between the umbra of $p 1$ and the unusual penumbra $n 1$ changes its polarity negative to positive. This is the effect of projection and occurs in all normal penumbrae. When the region is near the solar limb, the polarity of the penumbra is reversed because we look at the field lines from behind; as the region is rotated to the disk center, the polarity changes to its real sign. From the location of the region on June $28-29$, we estimate the inclination angle of the penumbral field lines to be between $30-40$ deg to the horizontal. This is an excellent way to measure the Wilson depression, and we plan a project on this soon.

\section{5. $\delta$ Spots}

The relationship between flare occurrence and magnetic fields has been studied by many authors, inter alia Švestka (1976) and Zirin and Liggett (1987). Evidence for magnetic flux cancellation has been given by Zirin (1984), Livi et al. (1989), and Wang et al. (1989). It has been established that flares only occur at neutral lines where gradients are steep and shears exist. These are most pronounced in the $\delta$ configuration, two umbrae of opposite polarity in the same penumbra. In large regions like this one, the 
$\delta$ configuration is easily recognized by minority or satellite flux, fields of the wrong polarity immersed in the regular fields.

While the overall structure of this region is simple and bipolar, there were two sites of $\delta$ configuration where almost all the flares occurred. The first site already existed when the active region appeared on the east limb, so we do not know the process of its formation. It consists of a leader spot ( $p 4)$ immersed (Figure 2$)$ in $f$ polarity. On June 27 it does not appear to be connected to any follower, but on June $29 f 7$ appeared (Figure 1), and clearly is connected to $p 4$ by penumbral fibrils in the frame for July 1. These two spots are part of the great penumbral complex including the umbrae $f 1, f 2$, and $f 4$ above it. Sheared penumbra separated $p 4$ from $f 1$. There is only a small amount of flux cancellation associated with this $\delta$ spot, and the size of umbrae does not appear to change throughout the observing run. Despite this, most of the larger flares had footpoints here. And some flares were limited to this area.

The second $\delta$ configuration consists of a pair of spots ( $p 3$ and $f 6$ ) from separate EFR's. Their evolution appears in Figures 4 and 5. $f 6$ was connected to $p 6$ but joined to $p 3$ which appeared overnight on June $27-28$ to form a $\delta$ spot. This is especially odd because $p 2$ was also nearby but made no connection to $f 6$; it simply moved ahead to join $p 6$. Why should one spot lock together with another when a second does not? One possibility is that the connections already exist below the surface.

At the beginning of June 28 , the umbrae $p 3$ and $f 6$ were very close. During the 28th the umbrae of the two spots steadily shrank, but they were always separated by a small light bridge about $700 \mathrm{~km}$ across. We observed 6 flares to begin there, presumably there were others during our night. In most cases we saw one footpoint just NE of the $\delta$ spot and the other footpoints elsewhere. But there is no question that virtually all the flares in the region were associated with the two $\delta$ configurations. By the 29th $p 3$ and $f 6$ had disappeared (Figure 2), but plage of both polarities remained which steadily cancelled in the course of the day, accompanied by more flares, the last at $22: 00$.

Total flux loss for this pair of $\delta$ spot was roughly $8 \times 10^{20} \mathrm{Mx}$, giving a flux cancellation rate of $10^{16} \mathrm{Mx} \mathrm{s}^{-1}$. If the fields were $10000 \mathrm{~km}$ deep, we get an energy of $10^{32} \mathrm{ergs}$, about the energy of the numerous modest flares. This is the first case we have observed of mutual disappearance of such substantial spots. However, we were not measuring vector fields at the time and cannot comment on the exact manner in which the flux disappearance took place.

\section{Flare Morphology}

All flares in this region occur at neutral lines associated with satellite polarity inclusions; half of these were the loci of prominent flow motions. Many of the initial kernels appeared in regions disturbed by the motion of the spot $p 5$. These flares were generally homologous, with footpoints in the same areas. However, it is striking that a flare occurring at one sheared neutral line will produce footpoints at other, more distant ones. These distant footpoints are almost always in the other flare-producing region. Figure 7, for example, shows a flare associated with $p 5$ that also has a footpoint near $p 4$, the other 
$\delta$ spot, at the other end of the region. The reason is unclear. Could there be a magnetic connection between all these inclusions?

Because we had videotapes of high time resolution, we were able to study whether the footpoints of the flare in $\mathrm{H} \alpha$ brightened simultaneously. They did not, and the average delay between the flash phases in the individual footpoints ranges from 40 to $160 \mathrm{~s}$, a surprisingly long time.

Since the purpose of this paper is to study the general relationship between the evolution of magnetic fields and flares, we do not intend to study every flare of this region in detail. However, we demonstrate 4 selected examples of flares in Figures 6-9. In each figure upper-left is $\mathrm{H} \alpha$ center line; upper-right, $\mathrm{H} \alpha+0.7 \AA$; lower-left magnetogram; and lower-right, D3 filtergram. Arrows marked on $\mathrm{H} \alpha+0.7 \AA$ and magnetogram frames show the footpoints of flares.

Figure 6 shows a small flare which occurred at 13:56 UT, June 28. Videotapes show the initial brightening to have occurred at the two arrows at lower left. The footpoints (arrows) at upper right brightened $40 \mathrm{~s}$ later. If we check the field at the arrows in the VMG, we see weak field only, contrary to all our previous assertions. Since the later

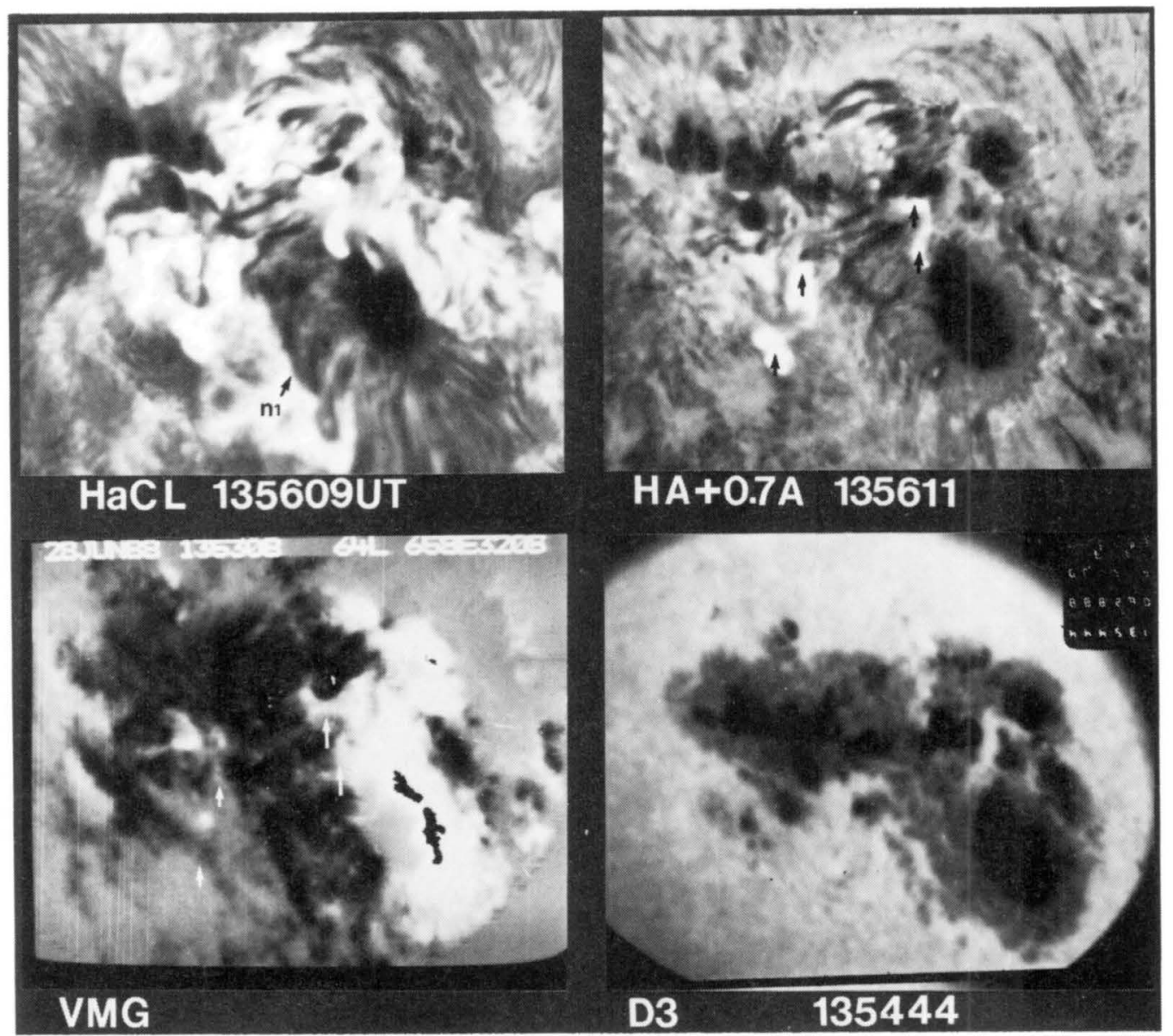

Fig. 6. Observations of a flare in three wavelengths plus a VMG. Videotapes show the initial brightening at the two arrows at lower left. The footpoints at upper right brightened $40 \mathrm{~s}$ later. Since the starting points show weak field only, it is possible that the true kernel of the flare was at the right but optical brightening was delayed. 
kernel at the right was associated with the $\delta$ spot, maybe the true source of the flare was at the right but optical brightening was delayed. Clearly we still have much to learn about this. In the $\mathrm{H} \alpha+0.7 \AA$ picture, arches are seen connecting the upper-right and upper-left footpoints. There was no D3 brightening for this flare.

Figure 7 shows a larger flare at 17:29 UT, June 28, which was a good example of the brightenings at the flare-producing sites. The D3 footpoints are seen at the edge of the $\delta$ spot $f 6$ and below the moving spot $p 5$. Arrows show the sites of these two footpoints. The first brightening appeared near the $\delta$ spot $f 6$. But there was also a remote brightening below the $\delta$ spot $p 4$. The delay of brightening of this footpoint is $160 \mathrm{~s}$.

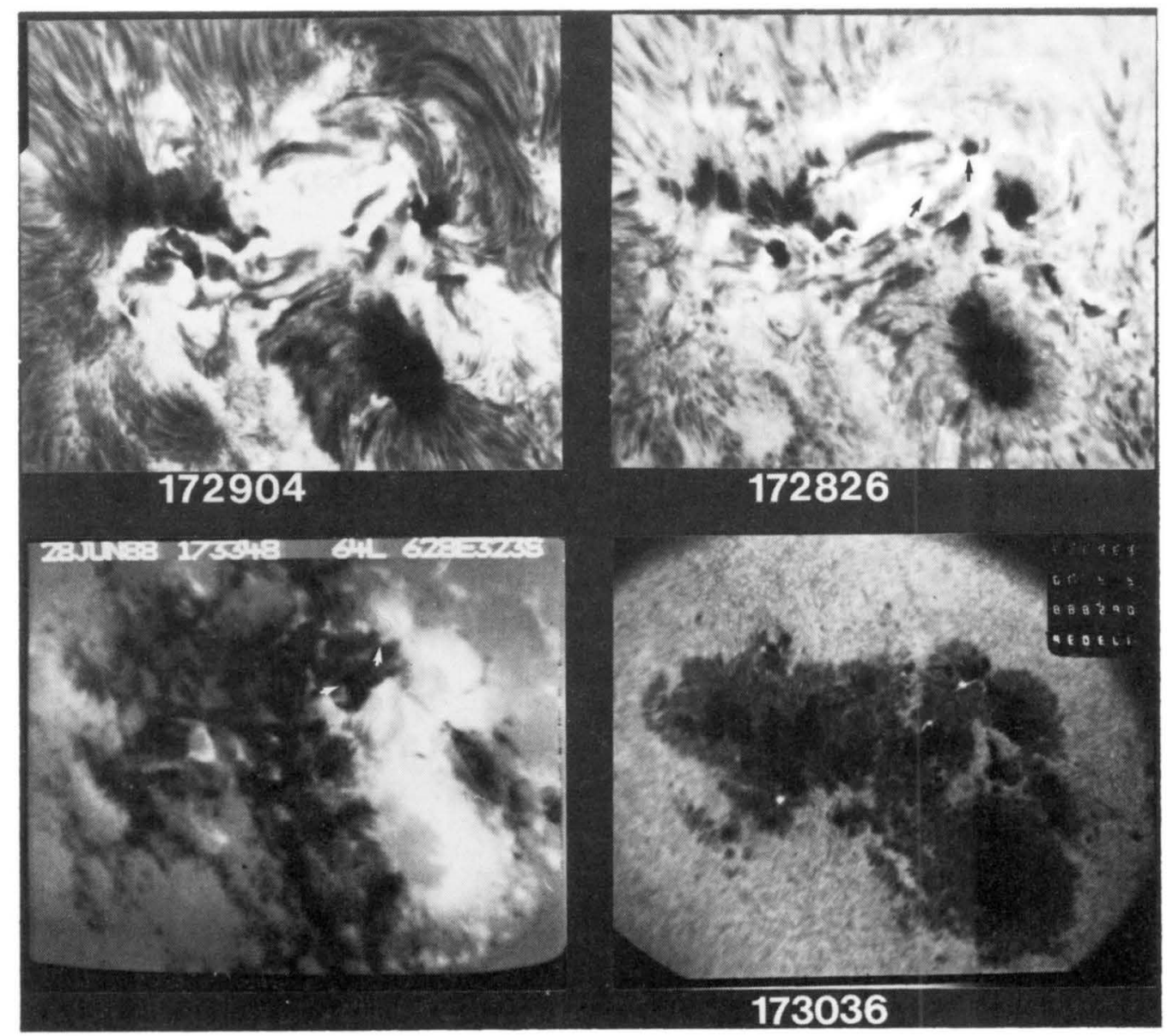

Fig. 7. Flare at $17: 29$ on June 28 in same wavelengths. The D3 footpoints are at the $\delta$ spot $p 3-f 6$, also at the rapid growing spot $p 5$. The first brightening appeared near the $\delta$ spot. Arrows show the sites of initial flare brightenings in $\mathrm{H} \alpha$.

Figure 8 shows a flare similar to that of Figure 7, with footpoints at $\delta$ spot $f 6$ and moving spot $p 5$, except that this time $p 5$ brightened first. This flare occurred at $01: 05$ UT, June 29. Remote brightening in $\mathrm{H} \alpha$ was also observed in the neutral line of another $\delta$ configuration $p 4-f 1, f 2, f 4$. But the only brightening in D3 was at the distant $\delta$ spot $p 4$.

Figure 9 shows the brightest flare that we observed in the region, which occurred at 


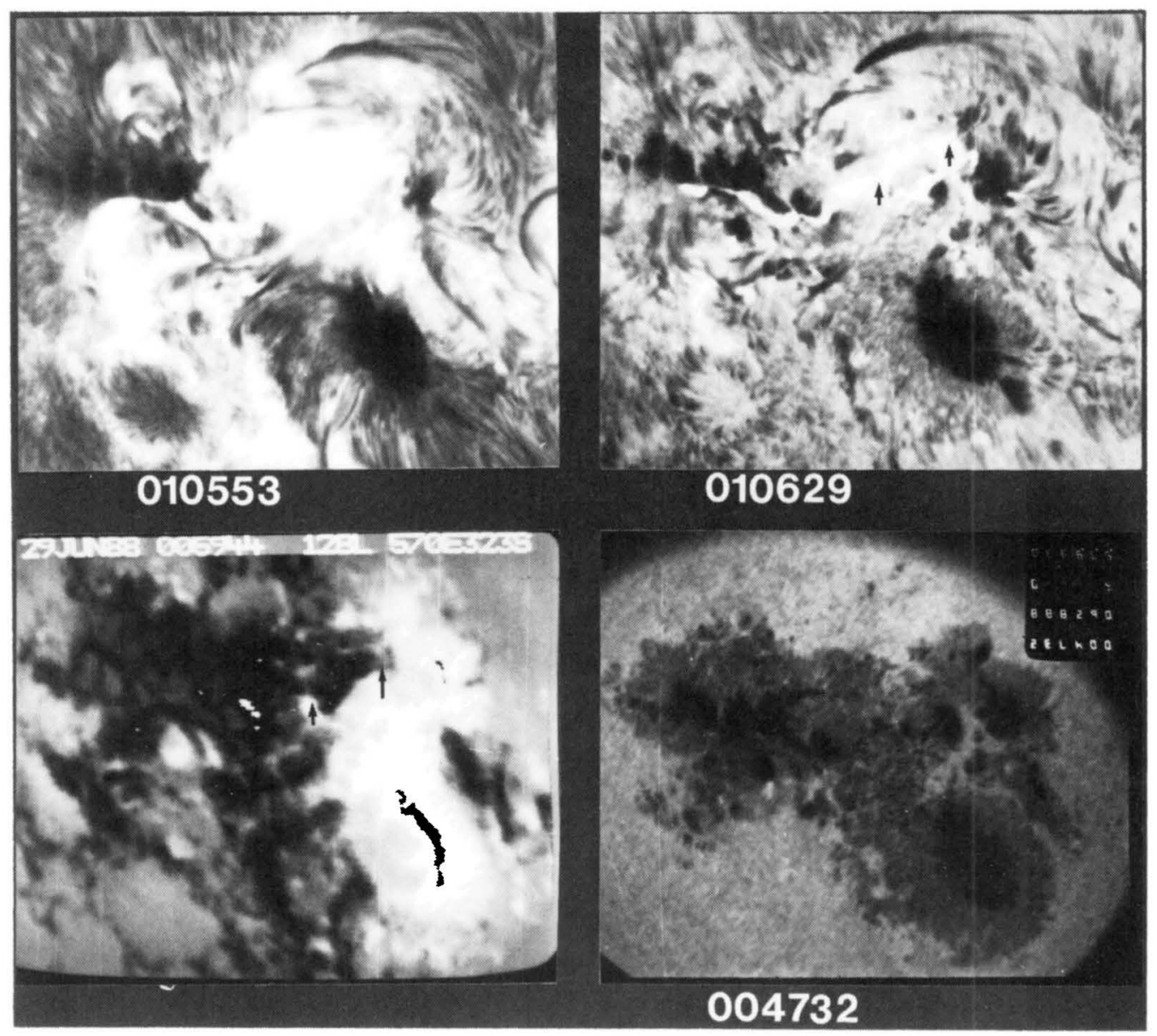

Fig. 8. A similar flare, with footpoints at $\delta$ spot and $p 5$, except that this time $p 5$ brightened first. Remote brightening below $f 1-f 2-f 4$ occurred 80 s later.

20: 16 UT, June 29. The first brightening occurred below $p 4$, a $\delta$ spot, and a nearby point $r 1$. The magnetic field at $r 1$ was negative (dark) on the other magnetograms, but during this flare it apparently reversed due to the reversal of the absorption line. The brightening at the right in the same region as earlier flares occurred about $40 \mathrm{~s}$ later. This brightening also filled the light bridge $p 2-p 6$.

In summary, almost all the flares belong to following two categories: (1) Flares initiated in the relatively stable $\delta$ spots $p 4-f 1, f 2, f 4$ at the left side of the field of view. (2) Flares initiated in the region disturbed by flux emergence, either at the short-lived $\delta$ spots $p 3-f 6$ or the rapidly growing spot $p 5$.

These flares are relatively small and do not show two-ribbon structures. In some cases, brightenings only occur on one side of the neutral lines; in other cases, the brightenings tend to occur right above the neutral lines. Although most of the flares are close to the $\delta$ spots, the footpoints usually are not located on the inversion line between the two spots.

The close relationship between flares and flows due to the new flux emergence is demonstrated statistically in Figure 10, which shows the number of flares in this region as a function of the dates. The data were quoted from the Solar Geophysical Data. Flare 


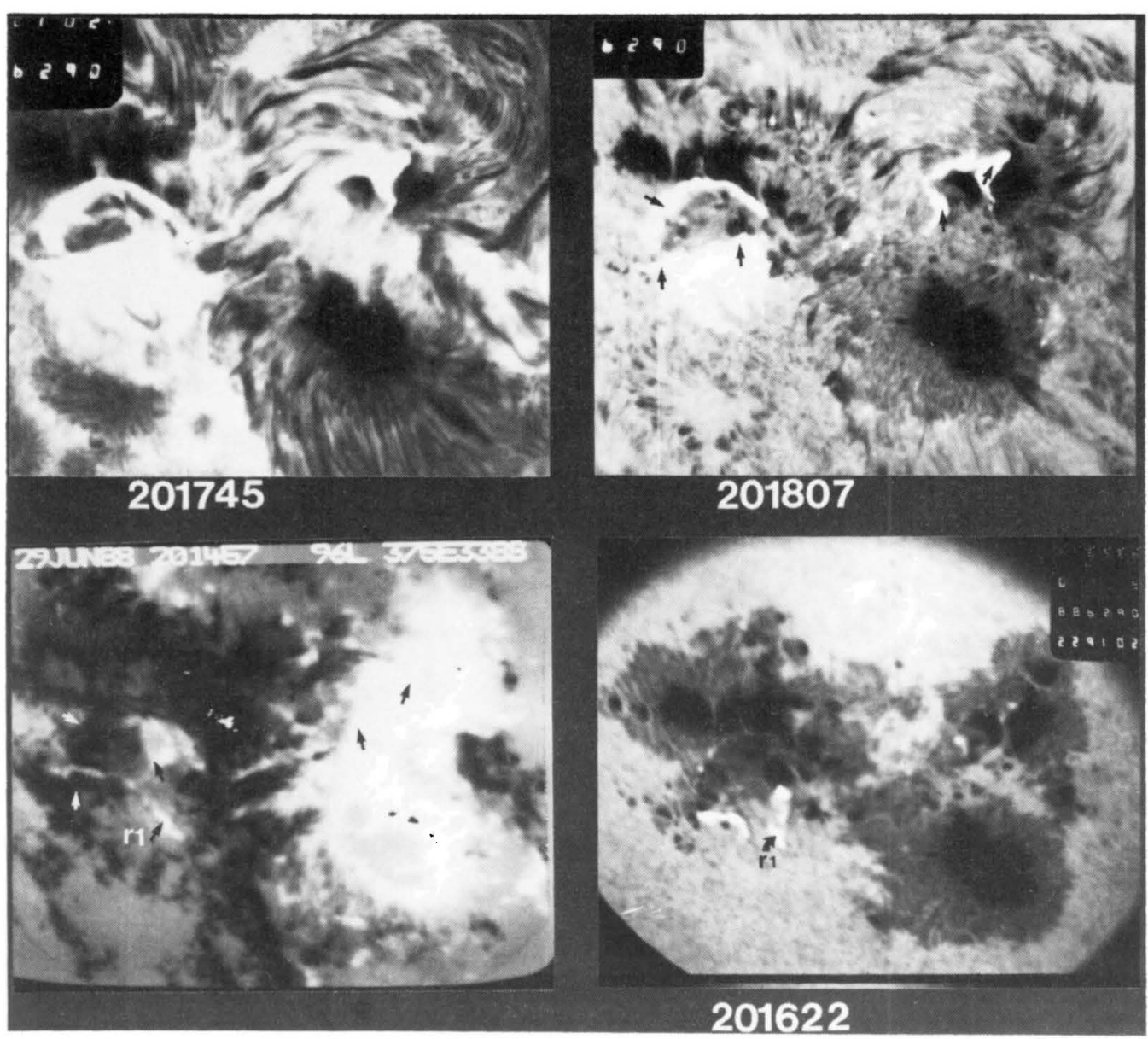

Fig. 9. The brightest flare in the region, which occurred at 20:16 UT, June 29. The first brightening occurred below $p 4$, the extreme right brightening $40 \mathrm{~s}$ later.

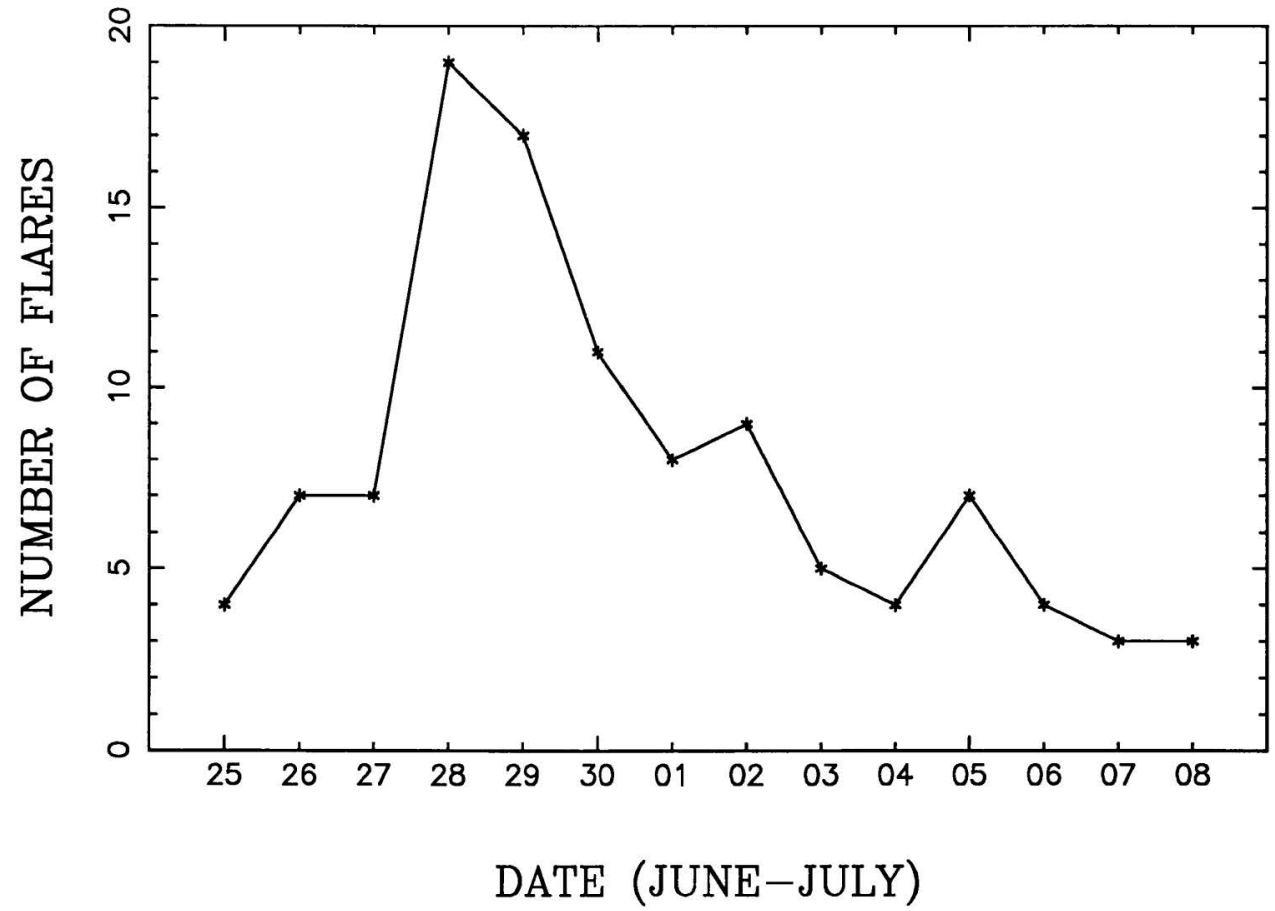

Fig. 10. Number of flares occurring in the active region BBSO No. 1167 during the period from June 25 to July 8,1988 . The number of flares peaked on June 28 , when the region showed the most prominent flow (C) Kluwer Academic Publishers - Provided by the NASA Astrophysics Data System 
number peaked on June 28 , when the region showed the most prominent flow motions due to the emergence of $p 5$ (Figure 1).

\section{Summary}

After studying the evolution of magnetic fields, the flares and flows, we may draw the following conclusions:

(1) Virtually all flares on July 28-29 in this region were related to either a relatively stable $\delta$ spot $p 4-f 1, f 2, f 4$ which has the obvious magnetic shear in their neutral line, or the area disturbed by the flows of newly emerging flux ( $\delta$ spot $p 3-f 6$ and rapid forming spot $p 5$ ).

(2) In the main area of flux emergence ( $p 5$ in Figure 3) the emerging magnetic polarities were not completely separated. Magnetic elements of leading polarity flowed westward through several elongated channels (ch1-3) in the following polarity. Negative flux was essentially fixed.

(3) Two end-to-end EFR's combined to produce a $\delta$ spot (Figure 2, f6 - p3) which disappeared completely in 24 hours, losing $8 \times 10^{20} \mathrm{Mx}$, i.e., $10^{32}$ ergs of energy. By contrast, another $\delta$ configuration changed very little during the whole observing period.

(4) We found a surprising decoupling of penumbrae from umbrae. In one EFR the umbra moves across penumbra to the front edge, and other small umbrae move right out of the penumbra. There were extensive penumbrae were not connected to any umbra (Figure 2, pp 1-2). These were due either to umbral flux returning to the surface, to flux left behind the moving umbra, or to pores that appeared and disappeared.

(5) We observed the merging of several pairs of leading spots of the same polarity. The spots did not merge completely, but were always separated by a thin light bridge. Similarly, the merging and cancelling $\delta$ spots were also always separated by a light bridge. Examining other data we find that all very large umbrae in the range $15000 \mathrm{~km}$ diameter are made up of smaller umbrae about $6000 \mathrm{~km}$ in diameter that can be distinguished in deep exposures.

\section{Acknowledgements}

We are indebted to the staff at BBSO for their assistance in obtaining the data. This work has been supported by NSF under grant ATM-8513577 and NASA under grant NGL 05002034.

\section{References}

Bray, R. J. and Loughead, R. E.: 1964, Sunspots, Chapman and Hall, London.

Danielson, R. E.: 1961, Astrophys. J. 134, 289.

Hale, G. E. and Nicholson, S. B.: 1938, Magnetic Observations of Sunspots, Carnegie Inst., Washington, p. 44. Livi, S. H. B., Martin, S. F., Wang, H., and Ai, G.: 1989, Solar Phys. 121, 197.

Mosher, J. M.: 1976, BBSO Preprint, No. 159.

Švestka, Z.: 1976, Solar Flares, D. Reidel Publ. Co., Dordrecht, Holland. 
Tarbell, T., Ferguson, S., Frank, Z., Title, A., and Topka, K.: 1988, Tenth Sacramento Peak Summer Workshop, Sunspot, NM.

Vrabec, D.: 1974, in R. G. Athay (ed.), 'Chromospheric Fine Structure', IAU Symp. 56, 201.

Wang, H. and Zirin, H.: 1988, Solar Phys. 115, 205.

Wang, H., Zirin, H., Patterson, A., Ai, G., and Zhang, H.: 1989, Astrophys. J. 343, 489.

Zirin, H.: 1984, Astrophys. J. 281, 884.

Zirin, H.: 1985, Australian J. Phys. 38, 961.

Zirin, H.: 1987, Solar Phys. 114, 239.

Zirin, H. and Liggett, M. A.: 1987, Solar Phys. 113, 267.

Zirin, H. and Tanaka, K.: 1981, Astrophys. J. 250, 791. 